\title{
A Comparison Among Distances Based on Neighborhood Sequences in Regular Grids
}

\author{
Benedek Nagy ${ }^{\star}$ \\ Department of Computer Science, \\ Faculty of Informatics, University of Debrecen, \\ PO Box 12. H-4010 Debrecen, Hungary, \\ Research Group on Mathematical Linguistics, \\ Rovira i Virgili University, Tarragona, Spain \\ nbenedek@inf .unideb.hu
}

\begin{abstract}
The theory of neighborhood sequences is applicable in many image-processing algorithms. The theory is well developed for the square grid. Recently there are some results for the hexagonal grid as well. In this paper, we are considering all the three regular grids in the plane. We show that there are some very essential differences occurring. On the triangular plane the distance has metric properties. The distances on the square and the hexagonal case may not meet the triangular inequality. There are non-symmetric distances on the hexagonal case. In addition, contrary to the other two grids, the distance can depend on the order of the initial elements of the neighborhood sequence. Moreover in the hexagonal grid it is possible that circles with different radii are the same (using different neighborhood sequences). On the square grid the circles with the same radius are in a well ordered set, but in the hexagonal case there can be non-comparable circles.
\end{abstract}

Keywords: Digital geometry, Neighborhood sequences, Square grid, Hexagonal grid, Triangular grid, Distance.

\section{Introduction}

The regular grids are very useful in many applications. They used in computer graphics and in image processing as well as in some applications of grid theory. In discrete mathematics they are called regular lattices. From both approaches there are well developed theories about this topic.

The theory of neighborhood sequences comes from the digital geometry. In [13. the two neighborhood relations are introduced on the square grid, and distance defined by each of them or by both of them (alternating used). In [2, 14 the periodic neighborhood sequences were investigated. The aim is to get more flexible distance functions in the digital plane. In 4 the authors introduced the

\footnotetext{
* This research was partly supported by the grant OTKA F043090.
} 
general, not necessary periodic neighborhood sequences. In [1] the neighborhood sequences in the cubic grid were analyzed.

The neighborhood relations are also well known, for the triangular and the hexagonal grid (they are used, for example in [3]). The neighborhood sequences based on these relations are defined for these grids in 9, 12. Note, that in this paper we refer the nodes of the grids as points, mostly for graphical reasons. In computer graphics and in description of networks this notion is usually used.

In this paper, after recalling the basic definitions and concepts we present some interesting differences among the distances on different grids. The simplest is the triangular grid, on which only 1 neighborhood relation and therefore only 1 distance can be defined based on the neighborhood relation. The square grid has huge literature, using two kinds of neighborhood relations. There are some recent papers about neighborhood sequences on the hexagonal grid (e.g. [11,12]). There are three kinds of neighbors, and two kinds of points (they have symmetric roles). Some surprising properties of the hexagonal grid is shown, in which there are significant difference between the hexagonal and the square (or triangular) grid.

\section{Definitions}

In this part we recall some definitions concerning neighborhood sequences. After the general approach the definitions are applied to the regular grids.

We can define the relation "k-neighborhood" among the nodes in (planar) graphs. Two nodes are neighbors if they are on the border of the same region. They are $k$-neighbors if they are neighbors and the shortest path between them includes at most $k$ edges.

It is obvious that these $k$-neighborhood relations are reflexive and symmetric relations. Moreover they have the following inclusion properties. All $(k-1)$ neighbors of a point are its $k$-neighbors as well.

According to the possible types of neighbors of a grid, we can define the so-called neighborhood sequences on this specific grid.

The infinite sequence $B=(b(i))_{i=1}^{\infty}$, - in which the values $b(i) \in \mathbb{N}$ are possible types of neighborhood criteria in the digital space that is used - is called a neighborhood sequence of the used grid.

Let $p$ and $q$ be two points (nodes) and $B=(b(i))_{i=1}^{\infty}$ a neighborhood sequence. A finite point sequence $\Pi(p, q ; B)$ of the form $p=p_{0}, p_{1}, \ldots, p_{m}=q$, where $p_{i-1}, p_{i}$ are $b(i)$-neighbor points (we call this step a $b(i)$-step) for $1 \leq i \leq m$, is called a $B$-path from $p$ to $q$. We write $m=|\Pi(p, q ; B)|$ for the length of the path. Denote by $\Pi^{*}(p, q ; B)$ a shortest path from $p$ to $q$, and set $d(p, q ; B)=$ $\left|\Pi^{*}(p, q ; B)\right|$. We call $d(p, q ; B)$ the $B$-distance from $p$ to $q$.

Now, let us see, how these definitions work for the regular grids.

\subsection{The Triangular Grid}

On the triangular grid there is only one neighborhood relation, it can be seen on the left hand side of Fig 1. The used coordinate system is a symmetric with three dependent (namely zero-sum) values (see right hand side of Fig. 1). 

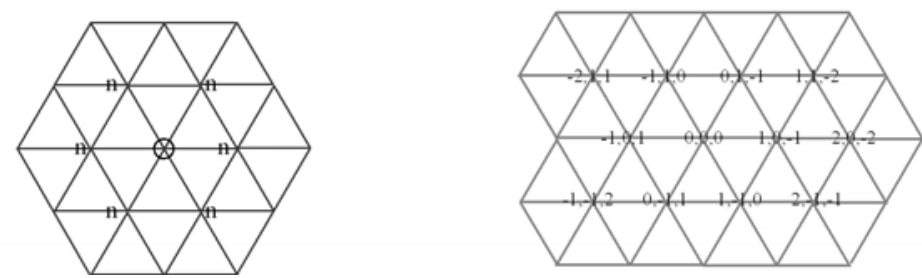

Fig. 1. The neighbors and the coordinate values of the triangular grid

By the help of the coordinate system we have a mathematical definition of the neighborhood: Let $p(p(1), p(2), p(3))$ and $q(q(1), q(2), q(3))$ be two points of the triangular grid. The points $p$ and $q$ are neighbors if the following condition holds:

1. $|p(i)-q(i)| \leq 1$ for $1 \leq i \leq 3$.

Since there is only one type of neighborhood, there is exactly one distance based on the neighborhood relation. Therefore the theory of neighborhood sequences in the triangular grid is almost trivial.

\subsection{The Square Grid}

The two neighborhood relations are from [13. A point has four 1-neighbors (excluding itself) and four more 2-neighbors. Fig. 2 shows both kinds of them (left). The used coordinate frame is the Cartesian (Fig. 2, right), which fits very well to this grid.
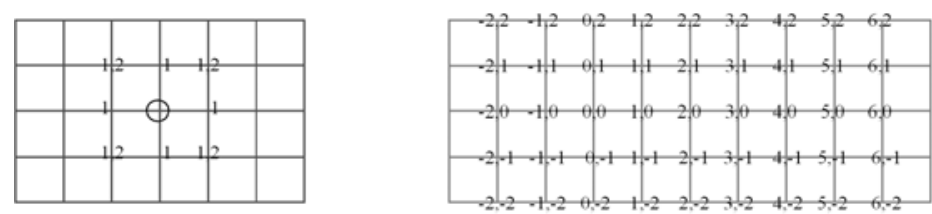

Fig. 2. Types of neighbors on the square grid and the Cartesian coordinate frame

So, mathematically one can redefine the neighborhood criteria in the following way: Let $p(p(1), p(2))$ and $q(q(1), q(2))$ be two points in $\mathbb{Z}^{2}$. Let $k \in\{1,2\}$. The points $p$ and $q$ are $k$-neighbors if the following two conditions hold:

1. $|p(i)-q(i)| \leq 1$ for $1 \leq i \leq 2$,

2. $\sum_{i=1}^{2}|p(i)-q(i)| \leq k$.

Because of the two kinds of neighbors, one can define various distances based on the neighborhood sequences (containing the values 1 and 2). 


\subsection{The Hexagonal Grid}

In this part we recall some important concepts about the hexagonal grid. Usually, we define three types of neighbors on this grid, as Fig. 3 shows on the left. Each point has three 1-neighbors, nine 2-neighbors (the 1-neighbors, and six more 2-neighbors), and twelve 3-neighbors (nine 2-neighbors, and three more 3 -neighbors). We use three coordinate values to represent the points of the grid (based on [1]), see Fig. 3, right-side.
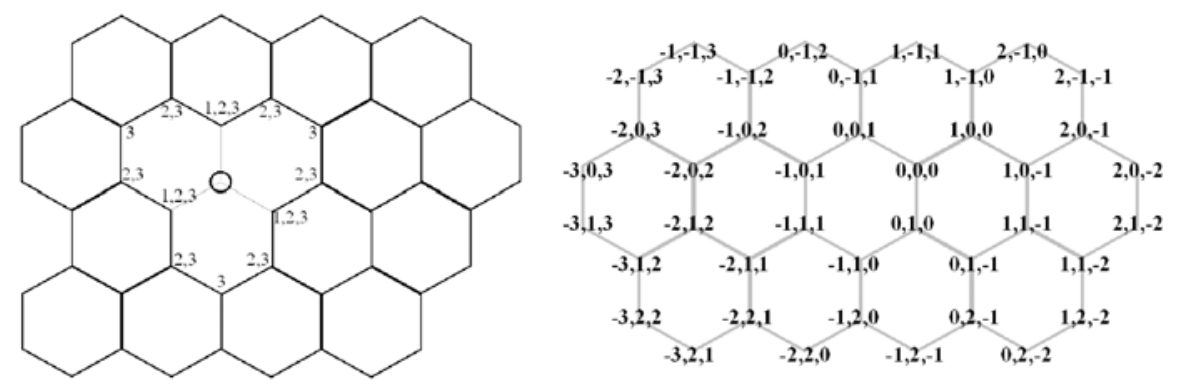

Fig. 3. Types of neighbors on the hexagonal grid and the coordinate values

We would like to give some other definitions and notation. We recall some of them from 19,11,12. We have two types of points according to the sum values of the coordinates. If the sum is 0 , then we call the point even, if the sum is 1 , then the point has odd parity.

We redefine the neighborhood relations mathematically using the presented coordinate-frame. Let $p(p(1), p(2), p(3))$ and $q(q(1), q(2), q(3))$ be two points in the hexagonal grid. Let $k \in\{1,2,3\}$. The points $p$ and $q$ are $k$-neighbors if the following two conditions hold:

1. $|p(i)-q(i)| \leq 1$ for $1 \leq i \leq 3$,

2. $\sum_{i=1}^{3}|p(i)-q(i)| \leq k$.

One can see that if two points are 1-neighbors, then their parities are different. Two non 1-neighbor, but 2-neighbor points have the same parities. Two 3 -neighbor points which are not 2-neighbors have different parities.

Since there are three different neighborhood relations on the hexagonal grid, there are more flexibility to define distances by neighborhood sequences.

\section{Properties of Distances}

It is obvious, that for all these distances the value is a natural number (number of steps). Moreover it is 0 if and only if the two points are the same. Now, we 
present some properties in which there are differences among the grids and the distances defined on them.

First let us show some properties of the distance on the triangular grid and a basic difference between the triangular grid and the others.

It is easy to show, the proofs can be found in 9,10 that the distance of two points $p(p(1), p(2), p(3))$ and $q(q(1), q(2), q(3))$ of the triangular grid is given by: $d(p, q ;$ tri $)=\max _{i=1,2,3}\{|p(i)-q(i)|\}$.

Note, that this distance has very nice properties. (It is very easy to compute, moreover:) It is close to the Euclidean distance. If the Euclidean length of a side of the triangle is 1 , and the digital distance of two points is $h$, then the Euclidean distance of them is between $\frac{\sqrt{3}}{2} h \approx 0.866 h$ and $h$.

The digital distance based on the neighborhood criterion of the triangular grid is a metric.

The property above does not hold for all distances based on neighborhood sequences on the square and on the hexagonal grid. To show this fact we present examples for both grids. Let $B=(2,1,1,1, \ldots)$ a neighborhood sequence. (It can be for both of the square and the hexagonal grid, because the 1-neighbors and the 2-neighbors are defined on both of them.) On square grid let $r(-1,0)$, $s(0,1)$ and $t(1,2)$ be three points. One can easily check that: $d(r, s ; B)=1$, $d(s, t ; B)=1$, but $d(r, t ; B)=3$. Therefore $d(r, s ; B)+d(s, t ; B)<d(r, t ; B)$.

Now, we are showing an example, when this $B$-distance fails on the triangle inequality on the hexagonal grid. Let $r(0,0,0), t(-2,1,1)$ and $s(-1,0,1)$ be three points on the hexagonal grid. One can check, that $d(r, s ; B)=1, d(s, t ; B)=1$ and $d(r, t ; B)=3$. Therefore we have $d(r, t ; B)+d(t, s ; B)<d(r, s ; B)$, again. A necessary and sufficient condition for the neighborhood sequence to generate a metrical distance is proved in [8] for the square grid and in [12] for the hexagonal grid.

Now we are dealing to another metric property, the symmetry.

On the square grid, all $B$-distances are symmetric. It is a simple consequence of the symmetry of the square-grid: all the points are of the same type, and with a central mirroring of the grid any two points can interchange each-other. In this way any shortest path has a mirror image path, which is also a shortest connecting the points in reverse order.

Now, we are presenting example for the interesting fact, that a $B$-distance can be non-symmetric on the hexagonal grid.

Let $p(1,0,-1)$ and $q(-1,1,0)$ be two points and $B=(3,1,1, \ldots)$ be a neighborhood sequence on the hexagonal grid. Then, by easy calculation one get: $d(p, q ; B)=2($ using $(0,1,0)$ at the first step). But, $d(q, p ; B)=3$. So $d(p, q ; B) \neq$ $d(q, p ; B)$. See Fig. 4 , where the shortest paths are shown for both directions.

This surprising fact occurs because there are two kinds of points, and we cannot use the symmetric pair of a path.

On square grid a $B$-distance $d(p, q ; B)>k$ never depends on the order of the first $k$ element of the used neighborhood sequence $B$. It is simple consequence of the fact that one can modify any of the coordinate values in any directions in each step (only the sum of the modified values are limited by the corresponding value 


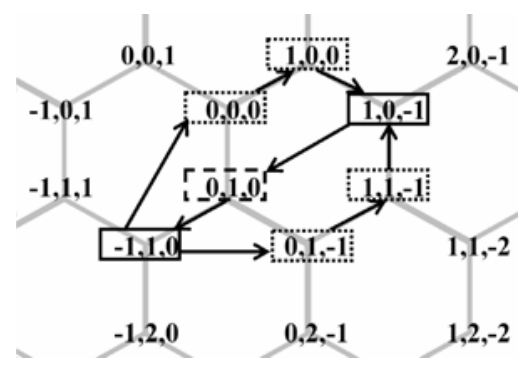

Fig. 4. The shortest paths between the points $(1,0,-1)$ and $(-1,1,0)$ using neighborhood sequence $(3,1,1, \ldots)$

of the neighborhood sequence). So one can use many mirroring transformations of the grid among the points of a path to reorder the elements of the neighborhood sequence getting a new path.

Opposite to this, in hexagonal grid we have:

Proposition 1. A distance $d(p, q ; B)>k$ can depend on the order of the first $k$ elements of $B$.

Proof. For proving this fact we need to show an example. Let our example be the following: let $p(1,0,-1), q(-2,2,1)$ and $B=(3,1,3,1,1, \ldots)$. Then $d(p, q ; B)=$ 3. Let interchange the first two elements of $B$ obtaining: $B^{\prime}=(1,3,3,1,1, \ldots)$. Then we have $d\left(p, q ; B^{\prime}\right)=5$. (Big difference!)

The fact above also related to the symmetric properties of the hexagonal grid and the two kinds of points.

Further in this section, we are detailing several other properties of the neighborhood sequences present on the hexagonal grid, but does not on the square (and on the triangular) grid.

In hexagonal grid, there are different neighborhood sequences which generate the same distance function, formally:

Proposition 2. There are different neighborhood sequences $B_{1}, B_{2}$ such that $d\left(p, q ; B_{1}\right)=d\left(p, q ; B_{2}\right)$ for all possible pairs of points $p, q$.

Proof. We show an example. Let $B_{1}=(3,3,3, \ldots)$ and $B_{2}=(3,2,2, \ldots)$ with

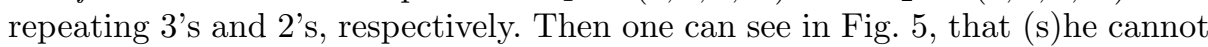
go further by 3 -steps than 2 -steps after the first 3 -step. Fig. 5 shows a point (black) with its 3-neighbors and with their 3-(or 2-)neighbors, since these sets are the same.

On square grid for each neighborhood sequence the generated distance function is different. We show, how one can find a point $p$ for each pair of $B_{1}$ - and $B_{2}$-distances (for $\left.B_{1} \neq B_{2}\right)$ such that $d\left((0,0), p ; B_{1}\right) \neq d\left((0,0), p ; B_{2}\right)$. Let $i$ be the smallest index with $b_{1}(i) \neq b_{2}(i)$. Let the first coordinate of $p$ be: $p(1)=i$ and the other one the larger number of the values 2 among the first $i$ element of 


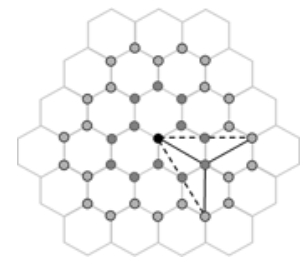

Fig. 5. The 3-neighbors (and non 2-neighbors) of a 3-neighbors (and non 2-neighbors) of a (black) point are 2-neighbors of one of its 2-neighbors

the sequences $B_{1}$ and $B_{2}$. Then the $B_{1}$-distance of the points cannot be the same as their $B_{2}$-distance, because the number of values 2 among the first $i$ elements differ for these neighborhood sequences. Using the neighborhood sequence containing more values 2 , the distance is exactly $i$, while with the other sequence the distance is $i+1$.

In the next proposition, we state that there are 'digital circles' on the hexagonal grid which are the same, but their radii is not equal (using different neighborhood sequences).

Proposition 3. On the hexagonal grid, there are neighborhood sequences $B_{1}$ and $B_{2}$ and there are different values $n, m \in \mathbb{N}$, such that the set of points for which $\left\{r \mid d\left(p, r ; B_{1}\right) \leq n\right\}$ and $\left\{r \mid d\left(p, r ; B_{2}\right) \leq m\right\}$ are the same with any point $p$.

Proof. We are presenting an example. Let $B_{1}=(1,1,1, \ldots), B_{2}=(2,2, \ldots), n=2$ and $m=1$. It is easy to check, that $\left\{r \mid d\left(p, r ; B_{1}\right) \leq n\right\}=\left\{r \mid d\left(p, r ; B_{2}\right) \leq m\right\}$ independently of the chosen point $p$.

It is trivial for the triangular grid that such a digital distance does not exist. On the square grid, it is also trivial. It is a simple consequence of the fact, that for instance the point $(n, 0)$ has exactly distance $n$ from the point $(0,0)$, independently of the used neighborhood sequence. (One can step 1 to left at each step, independently of the given value of the sequence.)

On the hexagonal grid, there is no way to go in only a single direction, the three coordinate values depend on each other. Only the distance of the 1-neighbors (including the point itself) of a point is independent of the used neighborhood sequence (in each other cases the neighborhood sequences $B_{1}=$ $(1)_{i=1}^{\infty}$ and $B_{2}=(2)_{i=1}^{\infty}$ define different values of distances). On the square grid, it is a simple fact, that the distances are independent of the neighborhood sequence, when the points has difference at most in 1 coordinate value. On the triangular grid there is no question, because only 1 distance is defined. (See Fig. [6.)

On the square grid the sets of the points which have distance at most $h$ from a fixed point $p$ are in a well ordered set. So, for each pair of neighborhood sequence $B_{1}$ and $B_{2}$ at least one of the following relations hold:

$$
\left\{r \mid d\left(p, r ; B_{1}\right) \leq h\right\} \subseteq\left\{r \mid d\left(p, r ; B_{2}\right) \leq h\right\}
$$



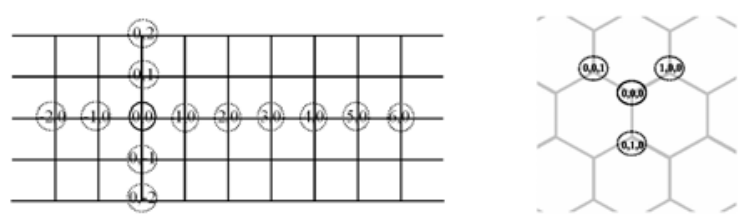

Fig. 6. The points for which their distance from the Origin are independent of the used neighborhood sequence

or

$$
\left\{r \mid d\left(p, r ; B_{1}\right) \leq h\right\} \supseteq\left\{r \mid d\left(p, r ; B_{2}\right) \leq h\right\}
$$

This ordering is exactly depends on the number 2's among the first $h$ elements of the neighborhood sequences. When these numbers are the same for two neighborhood sequences, then these sets are equal. If there are more values 2 in the initial part (up to index $h$ ) of $B_{1}$ than $B_{2}$ then the second set is a proper subset of the first one.

Now, we are presenting how this fact appears on the hexagonal grid. Let $r(0,0,0), s(-2,1,1), p(-1,2,-1)$ and $u(-1,2,0)$ be four points, and $B_{3}=$ $(3,1,2,2 \ldots), B_{4}=(1,3,2,2 \ldots)$ be two neighborhood sequences. Then one can compute, that:

$$
\begin{aligned}
& d\left(r, s ; B_{3}\right)=2<d\left(r, s ; B_{4}\right)=3, \\
& d\left(r, p ; B_{3}\right)=3>d\left(r, p ; B_{4}\right)=2, \text { and } \\
& d\left(r, u ; B_{3}\right)=2=d\left(r, u ; B_{4}\right) .
\end{aligned}
$$

So, one cannot say that the $B_{3}$-distances or the $B_{4}$-distances are greater than the other, it depends on the points as well. Moreover, the set of points which have 2 as $B_{3}$-distances from the point $(0,0,0)$ and the set of points which have 2 as $B_{4}$-distances from the point $(0,0,0)$ are non-comparable sets. So, the 'digital circles' with radius 2 using the origin $(0,0,0)$ are incomparable sets. It is also an important difference between the square and the hexagonal grids. We showed points which are in both of these sets (for instance $u$ ) and which are in the difference of them. (The point $s$ is in the first set, but not in the second, while $p$ is in the second set but it is not in the first one, see Fig. 7 .)

So, on the hexagonal grid there are $B$-distances and points such that:

- there are $B$-distances, value $h$ and points, such that from a point to another one the distances are the same (i.e. it is $h$ ) with the given neighborhood sequences,

- there are points such that for any chosen neighborhood sequence (among the used ones) the $B$-distance is $h$, but the other distance is greater than $h$.

This is also a surprising result, because on the square grid we have not this.

This difference occurs, because the symmetry of these grids, and because of the fact presented in Proposition 1 .

The facts described above are interesting properties of the hexagonal grid with comparison to the other regular grids. Now, we are showing a more strict property. 


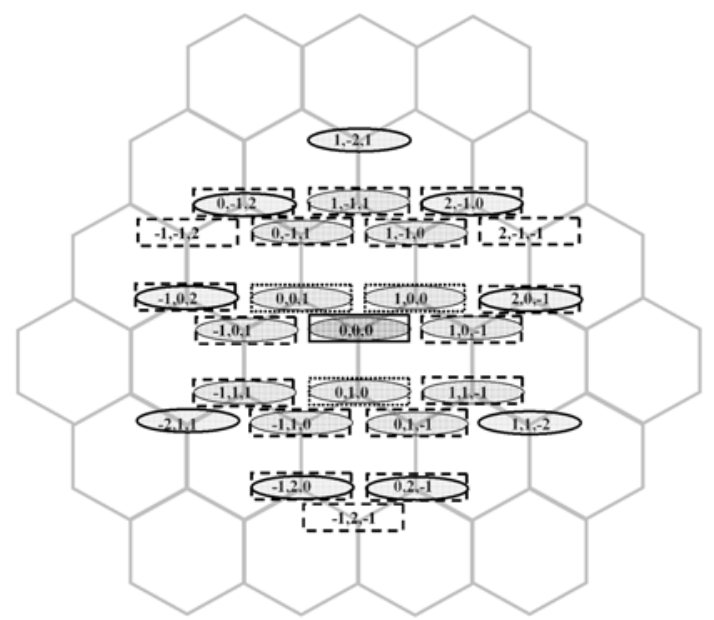

Fig. 7. The points of circles with radius 2 from the Origin by neighborhood sequences $(3,1, \ldots)$ (signed by ellipses) and $(1,3, \ldots)$ (signed by rectangles)

Proposition 4. On the hexagonal grid for every $h \in \mathbb{N}$ there are neighborhood sequences $B_{1}, B_{2}$ such that there are only a finite set $S_{h}$ of points has the next property: $d\left(p, q ; B_{2}\right)+h>d\left(p, q ; B_{1}\right)$ when $p$ is fixed and $q \in S_{h}$.

Before we prove this by an example, we can state it in a more general way:

Proposition 5. On the hexagonal grid there are neighborhood sequences $B_{1}, B_{2}$ such that for every $h \in \mathbb{N}$ and fixed points $p, r$ there are only a finite set $S_{h, p, r}$ of points has the next property: $d\left(p, q ; B_{2}\right)+h>d\left(r, q ; B_{1}\right)$ with $q \in S_{h, p, r}$.

Proof. Let $B_{1}=(1,1,1,1, \ldots)$ containing only 1's and $B_{2}$ contain $b_{2}(i)=2$ for all $i$. One can compute the set $S_{h, p, r}$ depending only on $h$ and on the points $p$ and $r$. It is a finite set, because the 'digital circle' using the neighborhood sequence $B_{2}$ is blowing faster than the circle using $B_{1}$.

It is trivial, that these facts above do not hold on the square grid.

As we have presented, the approximation of the Euclidean distance is good in the triangular grid. In [5] it is showed, that with neighborhood sequences one can obtain better approximation (but, with a lot more computing) on the square grid. The best approximations are given by neighborhood sequences on the hexagonal grid.

\section{Conclusions}

The square grid is well known and used in several applications. One of the advantages is that the Cartesian coordinate frame fits well to it. The triangular 
grid is simpler than the square grid, based on the widely used neighborhood criterion, only 1 digital distance can be introduced. When one wants a very fast and short computation with a quite good result, we recommend to use this grid instead of various neighborhood sequences on the square or on the hexagonal grid. The theory of neighborhood sequences is well developed for the square grid, but (with a little bit harder mathematic) on the hexagonal grid there are nicer results, according to the more flexibility (three kinds of neighbors). Some interesting properties of these distances are presented. The distance is an important concept in image processing. The distances presented here can have some non-usual applications as well. Non-metrical distances based on neighborhood sequences are used in practical applications in [6, 7].

\section{References}

1. P.E. Danielsson, 3D Octagonal Metrics, In: Proceedings of Eighth Scandinavian Conference on Image Processing 1993. pp. 727-736.

2. P.P. Das, P.P. Chakrabarti, and B.N. Chatterji, Distance functions in digital geometry, Information Science vol. 42, 1987, pp. 113-136.

3. E.S. Deutsch, Thinning algorithms on rectangular, hexagonal and triangular arrays, Communications of the ACM, vol. 15, no.3, 1972, pp. 827-837.

4. A. Fazekas, A. Hajdu and L. Hajdu, Lattice of generalized neighborhood sequences in $n \mathrm{D}$ and $\infty \mathrm{D}$, Publicationes Mathematicae Debrecen vol. 60, 2002, pp. 405-427.

5. A. Hajdu and B. Nagy, Approximating the Euclidean circle using neighbourhood sequences, In: Proceedings of the third KEPAF conference Domaszék, Hungary, Jan 2002, pp. 260-271.

6. A. Hajdu, B. Nagy and Z. Zörgö, Indexing and segmenting colour images using neighborhood sequences, In: Proceedings of IEEE International Conference on Image Processing, Barcelona, Spain, Sept 2003, vol 1, pp. 957-960.

7. A. Hajdu, J. Kormos, B. Nagy and Z. Zörgő, Choosing appropriate distance measurement in digital image segmentation, Ann. Univ. Sci. Budapest. Sect. Comput. vol. 24, 2004, 193-208.

8. B. Nagy, Distance functions based on neighbourhood sequences, Publicationes Mathematicae Debrecen vol. 63, 2003, pp. 483-493.

9. B. Nagy, Shortest Path in Triangular Grids with Neighborhood Sequences, Journal of Computing and Information Technology vol. 11, 2003, pp. 111-122.

10. B. Nagy, Metrics based on neighborhood sequences in triangular grids, Pure Mathematics and Applications vol. 13, 2002, pp. 259-274.

11. B. Nagy, A symmetric coordinate system for the hexagonal networks, In: Proceedings of Information Society 2004 - Theoretical Computer Science (ACM Slovenija conference), Ljubljana, Slovenia, Oct 2004, vol. D, pp. 181-184.

12. B. Nagy, Non-metrical distances on the hexagonal plane, In: Proceedings of the 7th International Conference on Pattern Recognition and Image Analysis: New Information Technologies, St. Petersburg, Russian Federation, Oct 2004, pp. 335338, accepted for publication in Pattern Recognition and Image Analysis

13. A. Rosenfeld and J.L. Pfaltz, Distance functions on digital pictures, Pattern Recognition vol. 1, 1968, pp. 33-61.

14. M. Yamashita and T. Ibaraki, Distances defined by neighborhood sequences, Pattern Recognition vol. 19, 1986, pp. 237-246. 